\title{
Router Designs for Elastic Buffer On-Chip Networks
}

\author{
George Michelogiannakis \\ Computer Systems Laboratory \\ Stanford University, Stanford CA 94305 \\ mihelog@cva.stanford.edu
}

\author{
William J. Dally \\ Computer Systems Laboratory \\ Stanford University, Stanford CA 94305 \\ dally@cva.stanford.edu
}

\begin{abstract}
This paper explores the design space of elastic buffer (EB) routers by evaluating three representative designs. We propose an enhanced two-stage EB router which maximizes throughput by achieving a $42 \%$ reduction in cycle time and $20 \%$ reduction in occupied area by using look-ahead routing and replacing the three-slot output EBs in the baseline router of [17] with two-slot EBs. We also propose a singlestage router which merges the two pipeline stages to avoid pipelining overhead. This design reduces zero-load latency by $24 \%$ compared to the enhanced two-stage router if both are operated at the same clock frequency; moreover, the single-stage router reduces the required energy per transferred bit and occupied area by $29 \%$ and $30 \%$ respectively, compared to the enhanced two-stage router. However, the cycle time of the enhanced two-stage router is $26 \%$ smaller than that of the single-stage router.
\end{abstract}

\section{Categories and Subject Descriptors}

B.4.3 [Hardware]: Input/output and data communicationsInterconnections; C.1.2 [Computer systems organization]: Multiple data stream architectures-Interconnection architectures

\section{General Terms}

On-chip networks

\section{INTRODUCTION}

Recent scaling of semiconductor technology enables many processing and storage elements to be integrated on a single die. Networks-on-chip (NoCs) provide a scalable communication infrastructure for such systems-on-chip [3,5]. As designs get larger, the effect NoCs have on the overall design increases. Thus, increasing network efficiency is essential.

This paper explores the design space of elastic buffer (EB) routers. EB routers are bufferless packet-switched routers. They have the area and energy benefits of circuit-switched

(C)ACM, (2009). This is the author's version of the work. It is posted here by permission of ACM for your personal use. Not for redistribution. The definite version was published in SC09, November 14-20, 2009, Portland, Oregon, USA. http://doi.acm.org/10.1445/1654059.1654062 routers, without the latency and cost overhead of setting up and tearing down circuits. They have significantly simpler designs because they lack virtual channels (VCs) [2], $\mathrm{VC}$ allocation, and credit logic. Furthermore, because each input may request at most one output, the switch is granted to inputs by output arbiters, instead of a complex allocator. EB routers operate by using master and slave latches of flipflops (FFs) as independent storage locations [17]. Therefore, the pipeline FFs in channels are used for buffering, in place of input buffers at routers. As a result of EB router design simplicity, the $5 \times 52 \mathrm{D}$ mesh $\mathrm{EB}$ router with dimension-order routing (DOR) presented in [17] has an $18 \%$ decrease in cycle time, $77 \%$ in area and $95 \%$ in dynamic power. This is compared to a similar VC router with 2 VCs with 8 buffer slots statically assigned to each, and buffers implemented as FF arrays.

We evaluate three representative EB router designs. The baseline two-stage router was presented in [17]. While simple, it requires a three-slot EB at each output to handle flow-control digits (flits) crossing the switch because arbitration is performed one cycle in advance and without credits. Moreover, routing and output arbitration are performed serially. Our first proposed design, the enhanced two-stage router, replaces the intermediate pipeline registers and output EBs with two-slot EBs to reduce cycle time and thus increase throughput in absolute time. A synchronization module maintains alignment between grants and flits. Moreover, look-ahead routing, first proposed in [7], is used so that output arbitration is performed in parallel with routing. Finally, our second proposed design, the single-stage router, merges the two stages of the enhanced two-stage router to avoid pipelining overhead and reduce router latency.

The enhanced two-stage router reduces cycle time by $42 \%$ compared to the baseline two-stage router. It also occupies $20 \%$ less area. The single-stage router occupies $30 \%$ less area and requires $29 \%$ less energy per transferred bit than the enhanced two-stage router. However, it has a $33 \%$ increased cycle time compared to the enhanced two-stage router. In our $8 \times 82 \mathrm{D}$ mesh and with each router operating at its maximum frequency, the single-stage router offers comparable ( $1 \%$ less) zero-load latency in absolute time compared to the enhanced two-stage router. Assuming an equal clock frequency, the enhanced two-stage router has a $32 \%$ increased zero-load latency compared to the single-stage router.

The optimal router choice depends on the clock frequency used for the routers. If all routers operate at the same clock frequency, the single-stage router is superior in terms of area and latency. If each router operates at its maximum fre- 
quency, the optimal choice for area is the enhanced twostage router. The baseline two-stage router provides the smallest energy per transferred bit. However, it is very close to the single-stage router which is preferrable in terms of cycle time, latency and area. The choice for designs prioritizing latency can depend on how the channel latency in clock cycles is affected by the clock frequency increase.

The rest of the paper is organized as follows: Section 2 outlines related work. Section 3 provides an overview of EB flow-control. Section 4 presents the three EB router designs in detail. Section 5 provides the evaluation methodology and results. Section 6 discusses the results and provides insights. Finally, section 7 concludes this paper.

\section{RELATED WORK}

The first router architectures explored for NoCs focused on VC flow-control [2]. Past work proposed look-ahead routing [7] and speculative allocation allowing allocators or router stages to be bypassed [10,20]. Express virtual channels [14] and token flow-control [13] allow the whole router pipeline to be bypassed. Further work has proposed using global lines to broadcast control information and arbitrate among different express virtual channels [12]. More optimizations have been explored such as dynamic VC buffer allocation and other aspects, such as fault-tolerance [9,21]. Asynchronous NoCs and router designs based on VC flowcontrol have also been proposed [24].

Routers for other flow-control schemes have been explored as well. Hybrid EB-VC networks using elastic channels in addition to input buffers at routers have been explored [11]. Other hybrid router schemes combining VCs or wormhole with circuit-switching by establishing a connection between frequent communication pairs have also been proposed $[6$, 18]. Routers with both best-effort and guaranteed traffic services have been investigated [23]. Furthermore, bufferless circuit-switched and packet-switched routers have been explored. Since flits cannot wait in the routers, they handle contention by emitting flits or packets in a non-ideal direction, also called deflection routing $[1,19,22]$, and can provide guaranteed throughput and multicast support [15]. In these networks, the topology is often the most influential factor on performance [16]. Alternatively, flits or packets under contention can be dropped and re-submitted by their source [8].

Our work extends previous work in that it performs a design space exploration of EB routers, by proposing and evaluating two new designs. Therefore, the gains EB networks offer in contrast to the currently-dominant VC flow-control are increased compared to [17].

\section{EB OVERVIEW}

EB flow-control uses the pipeline FFs in the channels for buffering. The addition of control logic to drive the latch enable pins of a master-slave FF separately enables their use as independent storage locations. Thus, each FF becomes an EB with two storage locations. The control logic can be implemented with a four-state finite state machine (FSM) [17]. EB channels feature multiple EBs to form a distributed FIFO. An EB is illustrated in Figure 1.

Flits advance to the next EB using a ready-valid handshake. An incoming ready $(\mathrm{R})$ signal indicates that the next $\mathrm{EB}$ has at least one free storage location to store an additional flit. An outgoing valid $(\mathrm{V})$ signal indicates that the

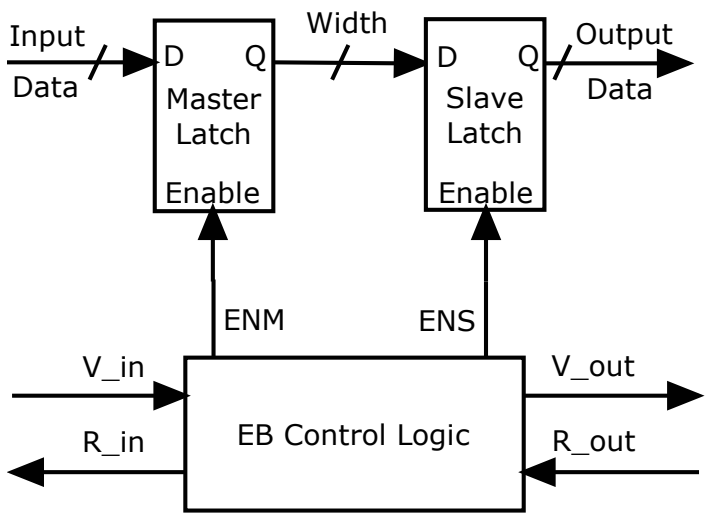

Figure 1: An elastic buffer.

flit currently being driven by the EB is valid. Flits advance when both ready and valid signals are asserted between two EBs at a rising clock edge. Since flow-control is applied on a per-flit basis, control logic is amortized over the width of the channel.

Using channels for buffering enables the removal of router input buffers. Removing router input buffers removes a significant part of the overall network energy and area costs. Moreover, this removes VCs [2] and credits from the network, compared to $\mathrm{VC}$ flow-control networks. In a router, this removes credit channel ports and logic as well as the VC allocator. Furthermore, it replaces the switch allocator with a switch arbiter for each output, since each input may request only one output. Because of design simplicity, the $5 \times 52 \mathrm{D}$ mesh EB baseline router with DOR presented in [17] has an $18 \%$ decrease in cycle time, $77 \%$ in area and $95 \%$ in dynamic power. These results are compared to a similar VC router with 2 VCs with 8 buffer slots statically assigned to each, and buffers implemented as FF arrays. A cell, gate and net comparison is included in Table 1 . The area and energy savings from the simplified router design are traded for an increased datapath width to compensate for the decreased channel utilization due to the removal of the input buffers.

The FIFO nature of the channels provides no isolation between traffic. Deadlock prevention is achieved by duplicating physical channels and preventing packets from being interleaved [17]. The latter is achieved by performing switch arbitration on a per-packet basis in EB routers.

\section{ROUTER ARCHITECTURE}

This section describes the three router designs evaluated in this paper. Section 4.1 outlines the baseline two-stage router of [17]. Section 4.2 describes the enhanced two-stage router. Finally, section 4.3 describes the single-stage router.

\subsection{Baseline Two-stage Router}

A block diagram of the baseline two-stage EB router of [17] is shown in Figure 2. Only one input and one output are illustrated in detail. The operation of this router is similar to that of a conventional packet-switched router. Incoming flits are stored into the input EB. The first pipeline stage consists of routing computation (RC) and switch arbitration (SA). A valid signal from the input $\mathrm{EB}$ indicates that a valid flit is driven in the first stage. The head flit's routing information 


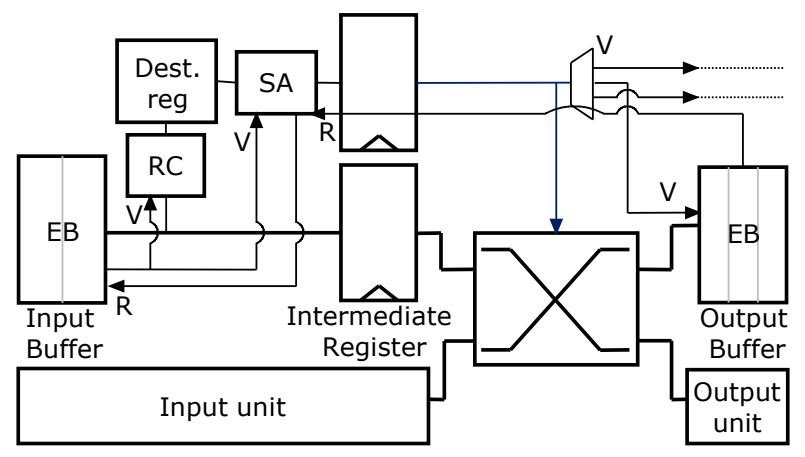

Figure 2: The baseline two-stage router.

goes through routing computation. Non-head flits of the same packet use the same selected output, stored in destination registers. When a flit receives a grant, it advances to the intermediate pipeline register. A ready signal is driven back to the input EB to release the flit. The switch arbiters temporarily de-assert their grant as long as their output EB is non-ready.

The second stage consists of switch traversal (ST) for flits which won arbitration in the previous cycle. Because arbitration is performed a cycle in advance of switch traversal (and without credits), an extra storage slot is required at the output EB to cover the pipeline delay. Each three-slot output EB de-asserts its ready output if it has two or more flits stored. The third slot is used to store any flit in transit when ready gets de-asserted. However, if an output EB contains two flits and its ready output was de-asserted during the previous cycle, there can be no flit in transit and it can therefore assert its ready output for one cycle. The output EB is implemented as a FIFO using a register file with rotating read and write pointers. Shift registers generate the read and write pointers pointing to one of the three storage locations, while combinational logic handles the rest of the output EB's inputs and outputs. Implementing the output EB as a three-slot EB would be more costly due to the complexity of the control logic to separately control three latches and handle all the cases of current and future flit locations.

The baseline two-stage router, while simple, has a long critical path in the first stage because routing and arbitration are performed serially. Moreover, the output EB introduces complexity and further area and energy overhead because it is implemented as a FIFO. This is an obstacle in meeting the output port timing constraints to drive long network channels.

\subsection{Enhanced Two-stage Router}

The enhanced two-stage router is illustrated in Figure 3. The intermediate register and output EB are replaced with two-slot EBs. This makes the output EB less costly in terms of area and energy and reduces its complexity giving tighter output port timing. It also allows using the intermediate EB for buffering, instead of only pipelining. This organization enables the router to operate with a smaller cycle time. The router employs look-ahead routing (LA R), first proposed in [7], to remove the routing computation from the critical path of the first stage. Head flits enter the first stage containing their selected output. Therefore, switch arbitration can begin as soon as the head flit arrives, without waiting

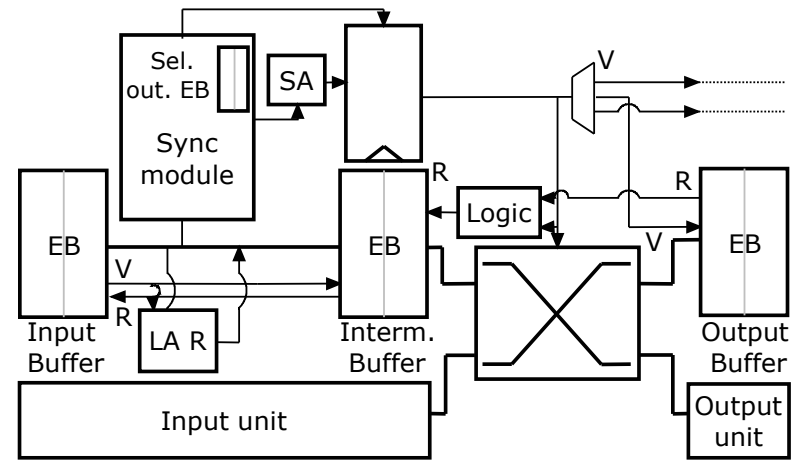

Figure 3: The enhanced two-stage router.

for routing computation. The look-ahead routing logic calculates the packet's output at the next hop and inserts it in the head flit via concatenation.

Flits advance to the intermediate EB even without receiving an output grant, as long as the intermediate EB is ready. Therefore, extra care must be taken to maintain alignment between flits arriving at the second stage and their grants. This is achieved by the synchronization module (sync module). It maintains the selected output ports for the flits stored in the intermediate EB in a separate selected output EB. The selected output port contained in the head of the selected output EB is always that of the current packet (oldest to have flits remaining in the first stage or the intermediate EB). The selected output EB may also contain in its tail (master latch) the selected output port of the next packet as shown in Figure 4 (left). In the worst case, only the tail of the current packet remains and there are two more single-flit packets. The most recent to arrive is contained in the intermediate $\mathrm{EB}$, and the other is driven in the router's first stage. In that case, the selected output EB stores the current packet's selected output port at its head and the next packet's selected output port at its tail. The third packet's selected output port is driven as an input. It will be enqueued when the third packet is enqueued into the intermediate EB, a cycle after the tail flit of the current packet departs.

The synchronization module detects when the tail of the current packet is about to depart from the intermediate EB and propagates the selected output port of the next packet to the switch arbiters. This is done one cycle in advance of the next packet being able to traverse the switch, as shown in Figure 5. Arbiter outputs are registered to shorten the critical path such that it does not extend past the router first stage. Thus, propagating the selected output port one cycle in advance is necessary to avoid inserting bubbles. Depending on the next packet's time of arrival, it may either have its head flit stored in the intermediate $\mathrm{EB}$, or driven in the first router stage. In the former case, the next packet's selected output port will be stored in the selected output EB's master latch. In the latter case, the selected output port will be driven as an input to the selected output EB. However, if the next packet's head flit has remained in the first stage for more than one cycle because the intermediate EB is full, the selected output EB is non-empty because there is no intervening packet, and the next packet's selected output port will be stored in the selected output EB. This is illustrated in Figure 4 (right). The synchronization mod- 


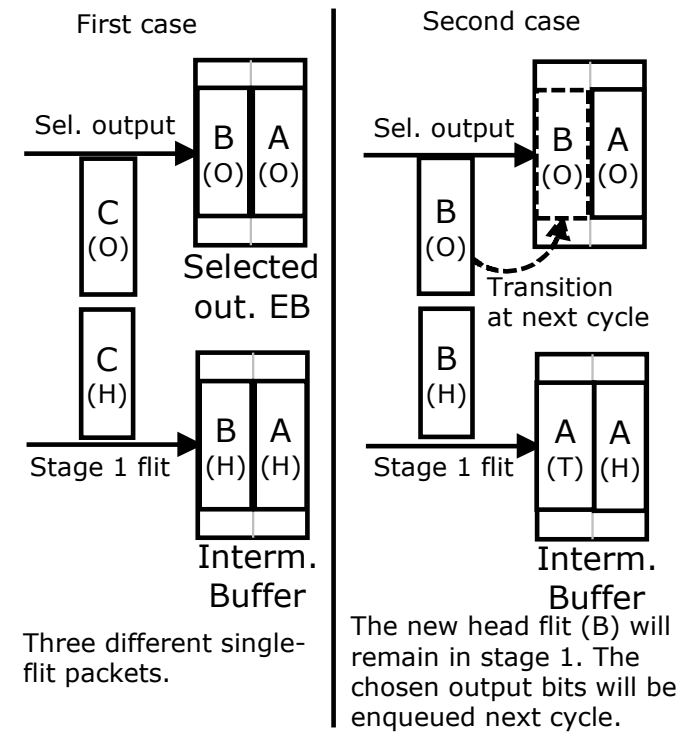

$\mathrm{H}$ : head flit. $\mathrm{T}$ : tail flit. O: selected output.

Figure 4: Synchronization module operation.

ule propagates the selected output port of the next packet from the the selected output EB's master latch, slave latch, or the input to the master latch, knowing if the intermediate and selected output EBs are full (non-ready) or empty (non-valid).

Flits at the head of the intermediate EB traverse the switch if they have a grant from their selected output and that output's EB is ready. Grants are made on packet boundaries and then gated by the selected output EB ready input. When a tail flit is traversing the switch, that input's synchronization logic asserts an update signal to all outputs. An output which receives an update signal from the input it is granting has its grant registers clocked at the next clock edge, thus updating the grants driven to the other router components. This is illustrated in Figure 5. Arbiters also have their grant register clocking enabled if they are currently granting no input, to assure that grants for newlyarrived packets will be propagated. An extra storage slot in the output EBs is not required because the decision to have the flit traverse the switch is made on the same cycle as it would arrive at the output EB.

\subsection{Single-stage Router}

The single-stage router is shown in Figure 6. It prioritizes latency instead of throughput and avoids pipelining overhead by merging the two stages of the enhanced two-stage router. Incoming flits request their selected output, calculated in advance by the previous router. Grants can only be given for outputs with ready output EBs. The grants from the switch arbiters enable the appropriate flits to traverse the switch and enter the output EBs. Look-ahead routing is performed in parallel, in the same manner as the enhanced two-stage router.

\section{EVALUATION}

The section presents evaluation results for the three EB router designs. Section 5.1 explains the evaluation methodology. Section 5.2 presents the results.

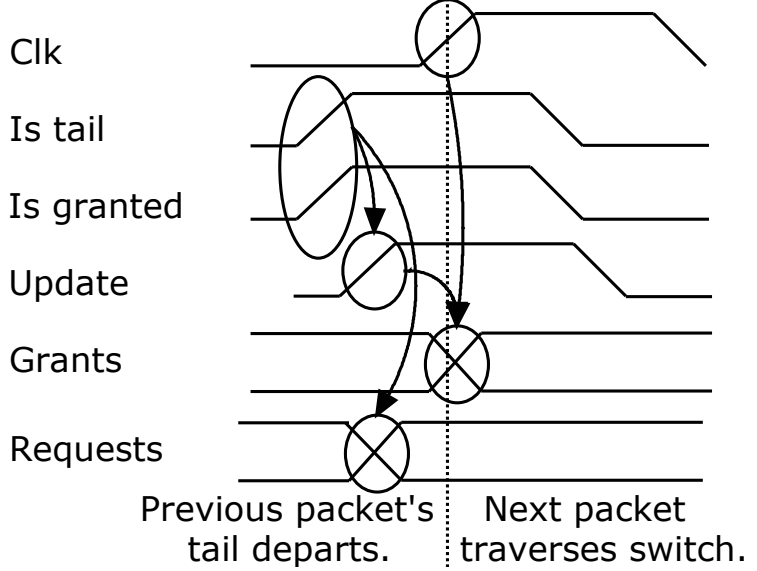

Figure 5: Updating output grants.

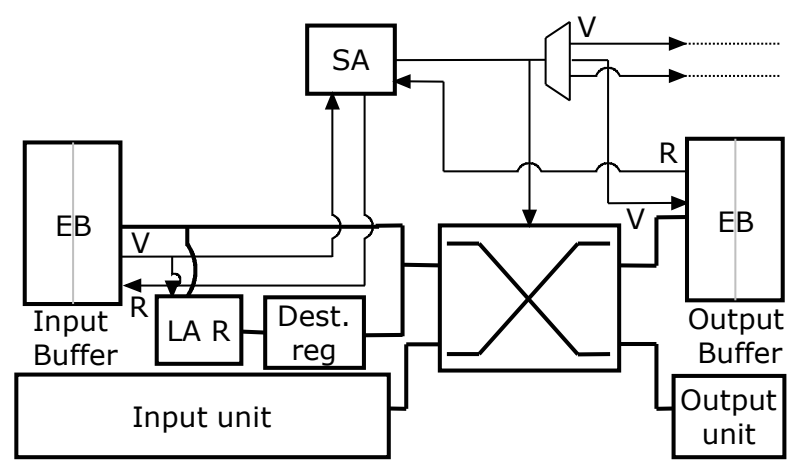

Figure 6: The single-stage router.

\subsection{Methodology}

Implementation results were obtained by synthesizing a single instance of each router design using Synopsys Design Compiler and placing and routing the synthesized netlists using Cadence Silicon Encounter. Clock frequencies were determined by static timing analysis using post place and route parasitics. Energy per transferred bit results were calculated by simulating the placed and routed netlists to record activity under an equal cycle time and flit injection rate for all cases, then multiplying by simulation time and dividing by the number of flits and flit size in bits.

We used a commercial 45nm low-power technology library under worst-case conditions. The initial floorplan utilization was set to $70 \%$. Primary input and output driving strengths, loads and timing constraints were specified to realistically assume network channels at the router ports. The network we assumed was an $8 \times 82 \mathrm{D}$ mesh using routers of radix 5 with a single network terminal attached to each router. Router ports were placed in the floorplan according to the inter-router connections of the assumed network. Deterministic DOR was used. Round-robin arbiters were used for switch arbitration. The switch was implemented using multiplexers.

The network throughput data points were generated using a modified version of Booksim [4] for EB networks. No communication protocol was assumed. Thus, there could be no protocol deadlocks. Therefore, we used a single physical 
network defining a single traffic class. We used the clock frequencies from the place and route results for each router and datapath width. For each router, one cycle was adequate for the flits to traverse our $2 \mathrm{~mm}$-long channels. Sources generate packets according to their injection rate. For all curves, the packet size was held constant at 512 bits. The datapath width was swept from 29 to 171 bits such that packets consisted of 3-18 flits. Flits are of the same width as the router datapath (they consist of 1 phit). Each cycle, up to one flit can be injected into the network from the injection buffer, and one ejected and stored into the ejection buffer.

To generate results for a variety of traffic patterns, we used a set of traffic patterns [4] for the throughput curves. That set consists of uniform random, random permutations, shuffle, bit complement, tornado and neighbor traffic. Results are averaged over the set of traffic patterns for each sample point of the curves. The maximum throughput is the average of the maximum throughputs of each traffic pattern. Percentage summaries were calculated by calculating the average distance between the sampling points of the routers under comparison, dividing by the normalized aspect, and averaging among all sampling points.

To illustrate the effect of routers operating under different clock frequencies, we also present throughput curves assuming an equal clock frequency for all routers. Curves assuming an equal frequency use a cycle time of $4.45 \mathrm{~ns}$, the maximum obtained from place and route results. However, the routers were still optimized for minimum cycle time in the place and route flow. Throughput and latency are measured in absolute time for curves using different cycle times for each sample point.

\subsection{Results}

Figure 7 presents place and route implementation results for the three routers. Curves with place and route results are not smooth because the software tools for the place and route flow use randomized algorithms with heuristics (such as simulated annealing) to perform optimizations on discreet values (such as cell sizing). The enhanced two-stage router has a cycle time reduced by $42 \%$ compared to the baseline two-stage router and $26 \%$ compared to the singlestage. The baseline two-stage router requires $9 \%$ less energy per transferred bit compared to the single-stage router and $35 \%$ less compared to the enhanced two-stage router. The trends shown in Figure 7(b) for energy per transferred bit remain the same when comparing against throughput, instead of datapath width. Finally, the single-stage router occupies $30 \%$ less area than the enhanced two-stage router, and $44 \%$ less than the baseline two-stage router.

Table 1 presents the cell, gate and net count for each router for the smallest and largest datapath widths we explored, as well as 64 bits. Results from [17] for a similar VC router with a 64-bit datapath, 2 VCs of 8 buffer slots statically assigned to each, and buffers implemented as FF arrays, are included for comparison. Figure 8 presents a breakdown of the gates and cells in router components, for each router design and a 64-bit datapath width. Results shown for arbiters as well as input, output and intermediate registers or EBs are a sum of all five router ports. For the enhanced two-stage router, the intermediate EBs bar includes the gates and cells for the synchronization module.

The cycle time of the baseline two-stage router is constrained by the first stage for all datapath widths. The en- hanced two-stage router is constrained by the second stage for datapaths of 64 bits or greater. This causes the linear increase of the enhanced two-stage router's cycle time for those datapath widths. For datapath widths smaller than 64 bits and greater than 47 bits the enhanced two-stage router is constrained by the input $\mathrm{EB}$ which could not meet the input port timing constraints. This is because of the increased fanout from the EB control logic to the latches. For smaller datapath widths, the enhanced two-stage router is constrained by the arbitration path in the first stage. The arbitration path which drives the switch enables (grants) is critical for the single-stage router.

To explore the importance of using look-ahead routing and replacing the three-slot output EBs when designing the enhanced two-stage router, we implemented an intermediate router design with look-ahead routing and three-slot output EBs. For a 64-bit datapath, this design was able to reach a cycle time of $2.3 \mathrm{~ns}$, compared to $3.8 \mathrm{~ns}$ of the baseline and $1.8 \mathrm{~ns}$ of the enhanced two-stage router. Lower cycle times were not achieved because the three-slot output EBs failed to meet the output port timing constraints.

Figure 9 shows the relationship between throughput and zero-load latency. For each datapath width, the average maximum throughput and the zero-load latency were sampled. If routers operate at their maximum frequencies, the single-stage router offers the lowest zero-load latency. The enhanced two-stage router has comparable (1\% increased) zero-load latency, whereas the baseline two-stage router has an increase of $46 \%$. Assuming all routers operate at a cycle time of $4.45 \mathrm{~ns}$, the single-stage router still offers the lowest zero-load latency, with the baseline-two stage router having a $32 \%$ increase and the enhanced two-stage router a $34 \%$ increase.

Figure 10 shows Pareto optimal curves associating average maximum throughput with occupied area when routers operate at their own minimum cycle time, or at an equal cycle time of 4.45ns. Points on these curves represent optimal design points. Comparison of one aspect by normalizing for the other is done by drawing a horizontal or vertical line on the graph. For minimum cycle times, the throughput per unit area percentage gain for the enhanced two-stage router is $2 \%$ compared to the single-stage router, and $160 \%$ compared to the baseline two-stage router. For equal cycle times, the single-stage router provides a $48 \%$ improvement compared to the enhanced two-stage router, and $114 \%$ compared to the baseline two-stage router.

\section{DISCUSSION}

The significantly reduced cycle time of the enhanced twostage router compared to the baseline is due to look-ahead routing and replacing the three-slot output EB with a twoslot EB. Using look-ahead routing had a greater impact on cycle time since the baseline two-stage router is constrained by the first stage. Using a two-slot output EB is necessary to achieve cycle times below $2.3 \mathrm{~ns}$ for 64 -bit datapaths, due to the output port timing constraints. Moreover, it also provides area and energy savings because of removing the third storage slot and the FIFO control logic complexity.

Even though both stages were merged into one for the single-stage router, it uses look-ahead routing allowing it to meet smaller cycle times than the baseline two-stage router. Moreover, the cycle time increases by less than a factor of two compared to the enhanced two-stage router because of 

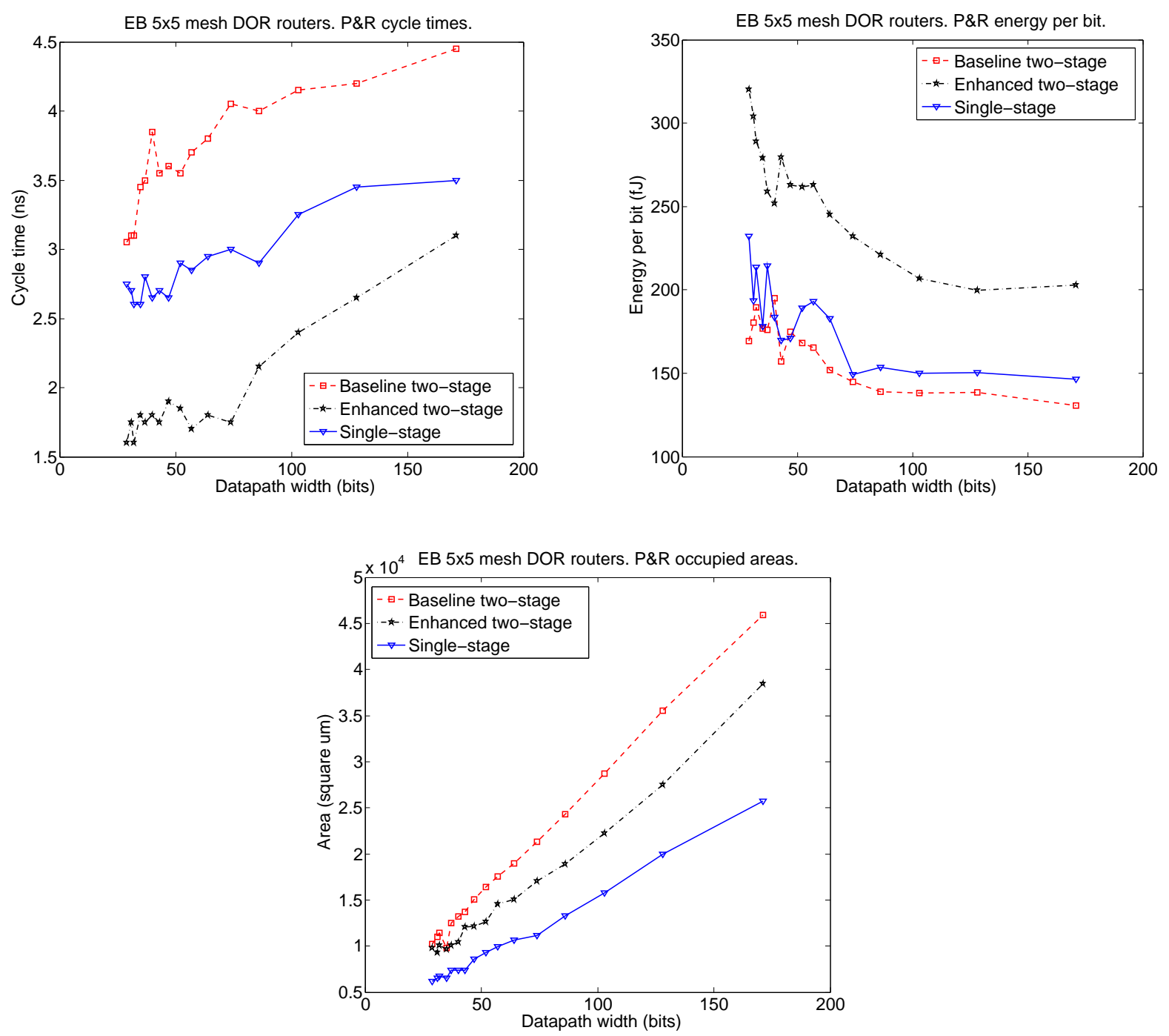

Figure 7: Place and route implementation results.

the lack of pipeline overhead.

As datapath width increases, the baseline two-stage router remains constrained by the first stage, indicating how large its critical path is. On the other hand, the enhanced twostage router is constrained by the first stage only for datapath widths smaller than 47 bits. This shows how much less complex the first stage of the enhanced two-stage router is, and also means that further optimization attempts should focus on other aspects of the router for larger data path widths. As the datapath width increases to very large numbers, the cycle times of all routers converge. This is because the switch dominates the cycle time for all three routers at these large widths, and all three routers use identical switches. Techniques such as switch splicing reduce the timing overhead of the second stage and thus will allow the enhanced two-stage router to be clocked at smaller cycle times for large datapath widths. However, routers with large critical paths on the first stage will have their cycle times unaffected.

The single-stage router occupies the least area. These sav- ings compared to the enhanced router are due to merging the two pipeline stages, thus removing the pipeline overhead. The enhanced two-stage router occupies less area than the baseline two-stage router because of the removal of the expensive three-slot output EBs. Since the synchronization module operates only on chosen output port bits, its significance is small compared to the datapath. Difference in cycle time has a small effect on occupied area because it only affects cell sizing and placement. Instead, the dominant factor is component complexity.

The enhanced two-stage router requires the most energy per transferred bit. This is attributed to the addition of the synchronization logic, splitting the intermediate register into two latches to implement the intermediate $\mathrm{EB}$, and to operating at an increased clock frequency — which forces the cells to have a greater driving strength. This affects cells in the whole router, including the switch. The single-stage router is much simpler than the enhanced two-stage router, reducing its energy consumption. However, it operates at a higher clock frequency which allows the baseline two-stage 
Table 1: Router implementation attributes.

\begin{tabular}{|c|c|c|c|c|c|c|c|}
\hline Aspect & Baseline & Enhanced & Percentage & Single-stage & Percentage & $\mathrm{VC}$ & Percentage \\
\hline & \multicolumn{7}{|c|}{29 bits } \\
\hline Gates & 9658 & 9228 & $-4.4 \%$ & 5817 & $-39.8 \%$ & & \\
\hline Cells & 2664 & 3712 & $+39.3 \%$ & 2522 & $-5.3 \%$ & & \\
\hline \multirow[t]{2}{*}{ Nets } & 2866 & 3506 & $+22.3 \%$ & 2388 & $-16.7 \%$ & & \\
\hline & \multicolumn{7}{|c|}{64 bits } \\
\hline Gates & 17930 & 14247 & $-21 \%$ & 10073 & $-44 \%$ & 60010 & $+235 \%$ \\
\hline Cells & 4837 & 6353 & $+31 \%$ & 4499 & $-7 \%$ & 15943 & $+230 \%$ \\
\hline \multirow[t]{2}{*}{ Nets } & 5082 & 5979 & $+18 \%$ & 4080 & $+18 \%$ & 16202 & $+219 \%$ \\
\hline & \multicolumn{7}{|c|}{171 bits } \\
\hline Gates & 43383 & 36343 & $-16.2 \%$ & 24313 & $-44 \%$ & & \\
\hline Cells & 11024 & 19190 & $+74.1 \%$ & 11088 & $0 \%$ & & \\
\hline Nets & 11887 & 14046 & $+18.1 \%$ & 10898 & $-0.1 \%$ & & \\
\hline
\end{tabular}

Gate count by router component (64-bit datapath)

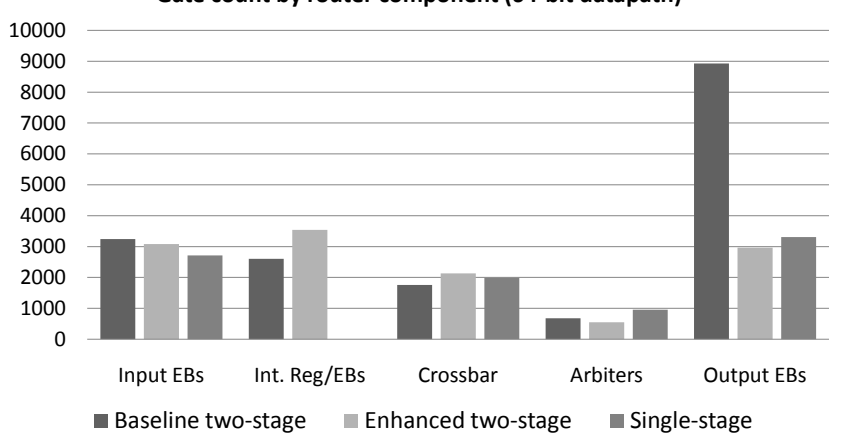

Cell count by router component (64-bit datapath)

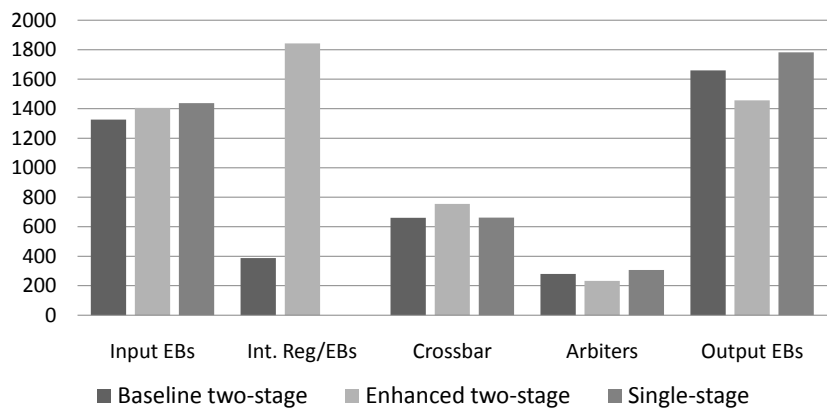

Figure 8: Router gate and cell breakdown.

router cells to be smaller. This is especially true for the switch, since it is not close to constraining the cycle time of the baseline two-stage router. This effect offsets the removal of the pipeline overhead.

The reduced complexity of the single-stage router is shown by its gate, cell and net count compared to the two-stage routers. The reduction in gates of the enhanced two-stage router compared to the baseline two-stage router reflects removing the FIFO logic of the three-slot output EBs. The increase in cells and nets is attributed to replacing the intermediate register cells with the intermediate EBs, which are made of two latch cells, connected with extra nets. This also makes the intermediate EBs larger in area, narrowing down the area savings compared to the baseline two-stage router.

Figure 8 illustrates that the three-slot output EBs of the baseline router have $175 \%$ more gates than the two-slot output EBs, showing how expensive the FIFO logic of the threeslot output EBs is compared to the control logic for two-slot EBs. However, the output EB cell count is practically not affected because the dominant portion is the datapath. Furthermore, the cell count of the intermediate EBs of the enhanced two-stage router includes the synchronization module logic, and thus has increased by $375 \%$ compared to the baseline two-stage router. Finally, the gate and cell count of the switch arbiters is low $(61 \%$ fewer gates than the muxbased crossbar), illustrating the low arbitration complexity of EB routers.

As shown in the latency-throughput curves of Figure 9(b), the single-stage router offers the smallest zero-load latency per unit throughput, on average. This is because of the one less clock cycle delay to traverse the router. Its effect is directly dependent on the average number of hops of our network, therefore especially important in our multihop 2D $8 \times 8$ mesh with DOR. However, the difference with the enhanced two-stage router significantly decreases when clocking each router at its maximum frequency, compared to clocking them at an equal frequency. This is due to applying the same cycle time to the network channels as the routers. Therefore, the network with the enhanced two-stage router also has higher-frequency channels, which have a lower latency in absolute time. However, these results rely on the channel latency in clock cycles remaining equal when increasing the clock frequency. While this is true for our low clock frequencies and physical channel lengths, other network settings might find that increasing the clock frequency also increases the channel latency in clock cycles. In that case, the single-stage router will provide an additional reduction in latency compared to the enhanced two-stage router because the latter will have its channel latency in clock cycles increased.

In both latency-throughput curves, the two-stage routers do not have an equal zero-load latency, because their throughput for a given datapath width differs. Thus, normalizing for 

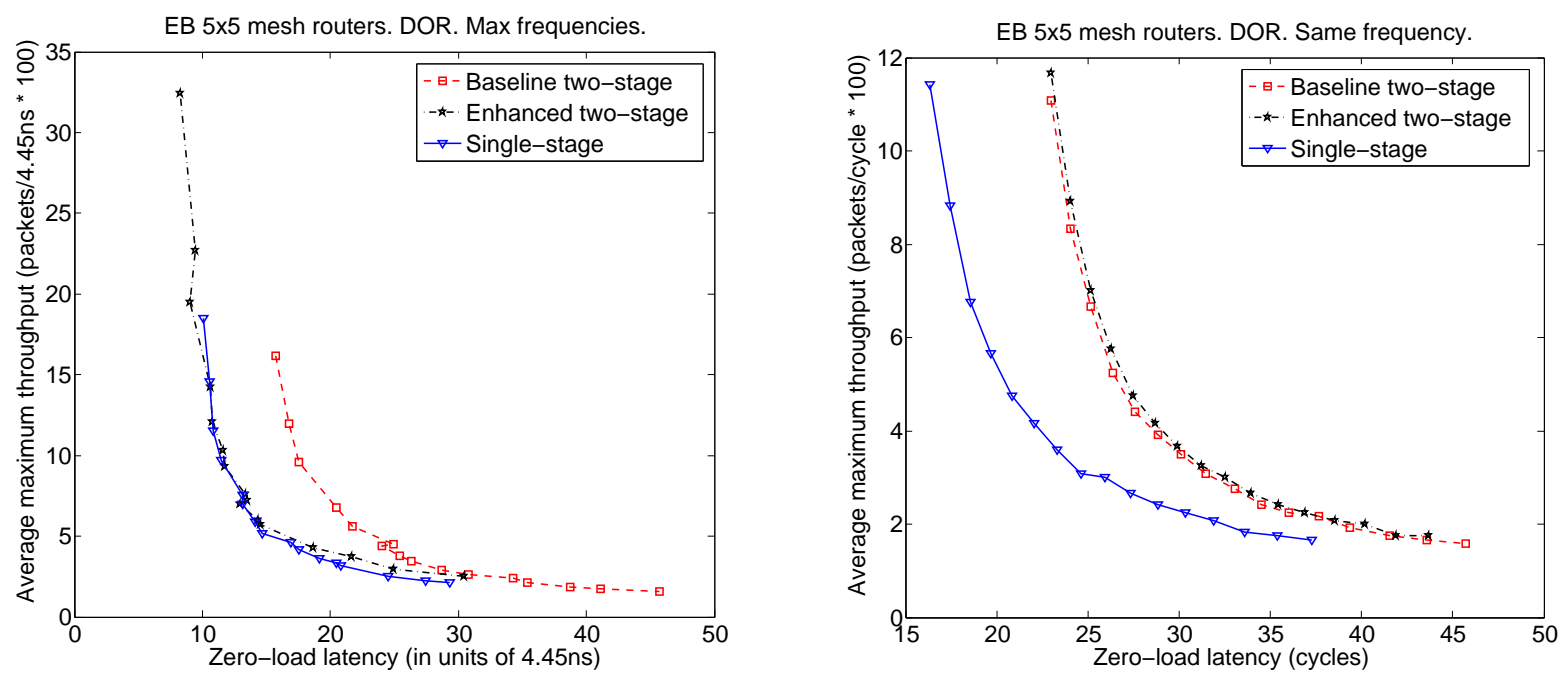

Figure 9: Latency-throughput comparison.

throughput uses unequal datapath widths, and thus different serialization latencies. Increasing the channel pipeline stages excessively has a diminishing return in terms of latency, because traversal time will be dominated by the pipeline overhead. Furthermore, the importance of router frequency will be reduced if we assume a network with a smaller average number of hops than our $8 \times 82 \mathrm{D}$ mesh. Such topologies will have their latency influenced more by the channel than by the router.

Since the routers were placed and routed for their maximum clock frequencies, their occupied areas remain the same regardless of the clock frequency they operate at. At an equal clock frequency, the single-stage router provides the most throughput per unit area because it occupies the least amount of area compared to the other two routers due to its simple design. Therefore, the single-stage router has an increased datapath width compared to two-stage routers occupying the same area. Thus, it can provide a higher throughput. However, if the three routers operate at their own maximum frequencies, the enhanced two-stage router provides more throughput per unit area due to its reduced cycle time.

The enhanced two-stage router is the optimal choice for networks prioritizing area. Designs prioritizing energy have the baseline two-stage router as their best choice, on average. However, it is closely followed by the single-stage router which carries cycle time, latency and area benefits. Designs with zero-load latency in mind should take into account the average number of hops and the effect on channel latency in clock cycles when applying the enhanced two-stage router's maximum clock frequency. Network designs which would clock all three routers under the same frequency have the single-stage router as their optimal choice in terms of area and latency. Examples of such designs can be systems-onchip, which may not require a higher clock frequency or may keep the network clock synchronized to a slower system-wide clock to avoid multiple clock domains. Table 2 summarizes which router is the optimal choice depending on design priorities.
Table 2: Optimal router choice for our $8 \times 82 \mathrm{D}$ mesh depending on design priority.

\begin{tabular}{|l||c|}
\hline \multicolumn{1}{|c||}{ Priority } & Router choice \\
\hline \hline \multicolumn{2}{|c|}{ Operate at maximum frequencies } \\
\hline \hline Area & Enhanced two-stage \\
\hline Energy & $\begin{array}{c}\text { Baseline two-stage } \\
\text { (closely followed by single-stage) }\end{array}$ \\
\hline Latency & $\begin{array}{c}\text { Single-stage } \\
\text { (depends on effect on channels) }\end{array}$ \\
\hline \hline \multicolumn{2}{|c|}{ Operate at the same frequency } \\
\hline \hline Area & Single-stage \\
\hline Energy & $\begin{array}{c}\text { Baseline two-stage } \\
\text { (closely followed by single-stage) }\end{array}$ \\
\hline Latency & Single-stage \\
\hline
\end{tabular}

The two new routers presented in this paper exemplify designing for different goals. Reducing the cycle time is important when designing for throughput. Thus, the most important cycle time constraints need to be identified and mended in the new design. On the other hand, router pipeline stages are a primary contributor to latency. Therefore, an improved design for latency can look into merging pipeline stages or be able to bypass them. Side effects of the designs, such as the channel cycle time or cost savings which can be traded for an increased datapath width, must also be investigated.

Further techniques can be applied to EB routers. For instance, speculation can be applied to bypass the first stage of a router, in a similar fashion as [20]. Output arbiters can grant an empty input if no input is requesting that output. If a flit arrives at the granted input, it can be written directly to the intermediate EB instead of the input EB and traverse the switch during the next cycle. This requires look-ahead routing to be performed at a different part of the router than the first stage. Furthermore, look-ahead 

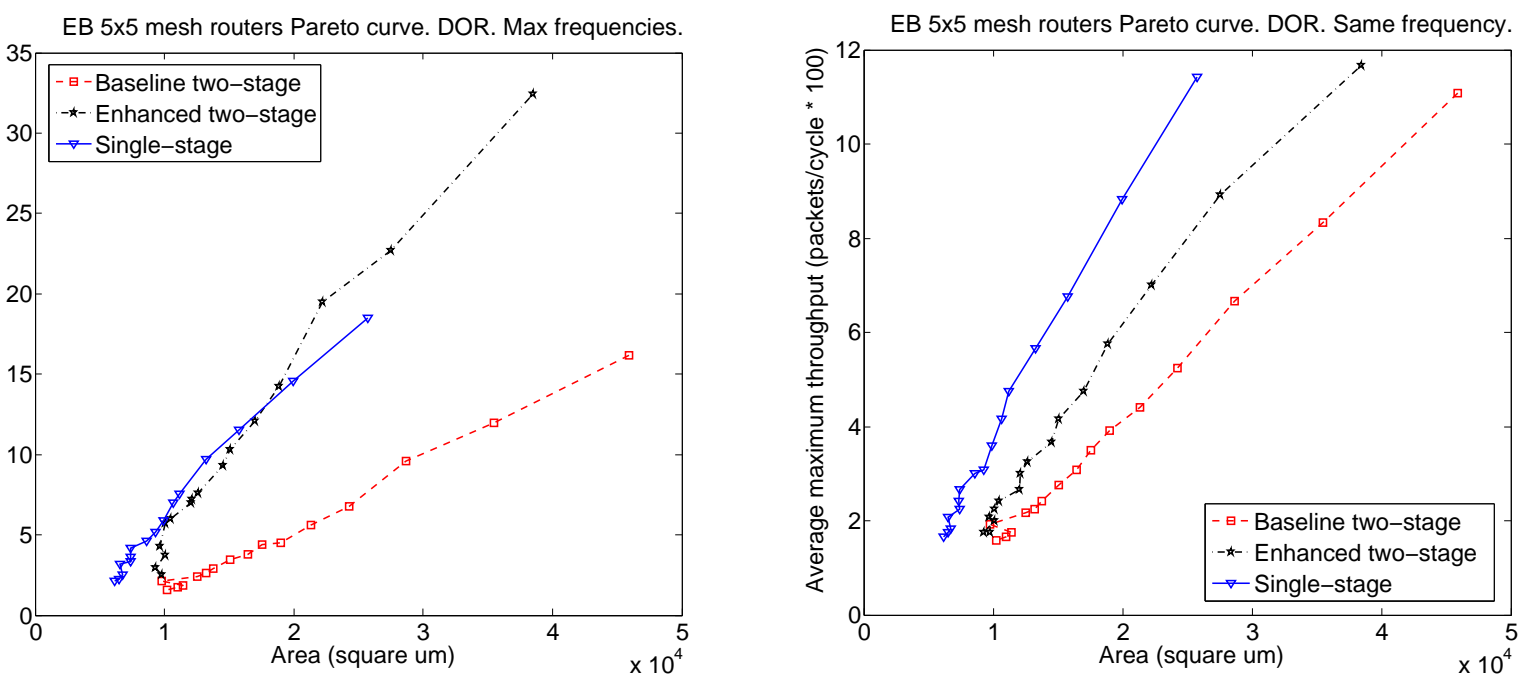

Figure 10: Throughput-area Pareto optimal curves.

adaptive routing algorithms may need extra mechanisms to sample network state at the router for which they are making the routing decision. Finally, router designs with clock cycle, energy or area savings can trade those savings for an increased datapath width.

\section{CONCLUSION}

This work presented three EB router designs representative of the design space: the baseline two-stage router, the enhanced two-stage router, and the single-stage router. The enhanced two-stage router replaces the three-slot output EB of the baseline two-stage router with a two-slot EB and uses look-ahead routing. These modifications optimize throughput reducing cycle time by $42 \%$ and area by $20 \%$. The baseline two-stage router's cycle time is always constrained by the first stage, whereas the enhanced two-stage router is constrained by the second stage for datapath widths of 64 bits or greater.

The single-stage router reduces router latency by merging the two stages of the enhanced two-stage router, also avoiding the pipelining overhead. This design reduces cycle time by $22 \%$ and area by $44 \%$ compared to the baseline two-stage router. Fusing the two pipeline stages gives a $33 \%$ longer cycle time than the enhanced two-stage router. With an equal clock frequency, an $8 \times 82 \mathrm{D}$ mesh with DOR using the enhanced two-stage router has a $34 \%$ increase in latency compared to the single-stage router $(32 \%$ using the baseline two-stage router). The single-stage router requires $9 \%$ more energy per transferred bit than the baseline two-stage router. Lastly, it offers comparable (1\% decreased) zeroload latency compared to the enhanced two-stage router if routers are clocked at their maximum clock frequencies, and $34 \%$ less latency if the routers are operated at the same frequency. Savings of a router design in area, energy or cycle time can be traded for a wider datapath.

The single-stage router is the optimal choice in terms of area and latency if all three routers would be clocked at the same frequency. Otherwise, the enhanced two-stage router is the optimal choice for network designs prioritizing area.
The baseline two-stage router provides the smallest energy per transferred bit. However, it is very close to the singlestage router which is preferrable in terms of cycle time, latency and area. Finally, if prioritizing for latency, the choice between the single-stage router and the enhanced two-stage router can depend on how channel latency in clock cycles is affected by the increase in clock frequency.

$\mathrm{EB}$ routers are simple, lacking allocation, credit and other overhead introduced by the currently-dominant VC flowcontrol [17]. Simple designs can be clocked at higher clock frequencies and provide area and energy savings. Thus, they can provide significant savings for many applications.

\section{ACKNOWLEDGMENTS}

This work was supported in part by the National Science Foundation under Grant CCF-0702341, in part by the National Security Agency under Contract H98230-08-C-0272 and in part by the Robert Bosch Stanford Graduate Fellowship.

\section{REFERENCES}

[1] P. Baran. On distributed communication networks. In IEEE Transactions on communication systems, pages $1-9,1964$.

[2] W. J. Dally. Virtual-channel flow control. IEEE Transactions on Parallel and Distributed Systems, 3(2):194-205, 1992.

[3] W. J. Dally and B. Towles. Route packets, not wires: On-chip interconnection networks. In $D A C$ '01: Proceedings of the 38th Conference on Design Automation, pages 684-689, 2001.

[4] W. J. Dally and B. Towles. Principles and Practices of Interconnection Networks. Morgan Kaufmann Publishers Inc., San Francisco, CA, USA, 2003.

[5] G. de Micheli and L. Benini. Networks on chip: A new paradigm for systems on chip design. In DATE '02: Proceedings of the conference on Design, Automation and Test in Europe, page 418, 2002. 
[6] J. Duato, P. Lopez, F. Silla, and S. Yalamanchili. A high performance router architecture for interconnection networks. In ICPP '96: Proceedings of the International Conference on Parallel Processing, Vol. 1, pages 61-68, 1996.

[7] M. Galles. Spider: A high-speed network interconnect. IEEE Micro, 17(1):34-39, 1997.

[8] C. Gómez, M. E. Gómez, P. López, and J. Duato. Reducing packet dropping in a bufferless NoC. In Euro-Par '08: Proceedings of the 14th international Euro-Par conference on Parallel Processing, pages 899-909, 2008.

[9] J. Kim, C. Nicopoulos, and D. Park. A gracefully degrading and energy-efficient modular router architecture for on-chip networks. SIGARCH Computer Architecture News, 34(2):4-15, 2006.

[10] J. Kim, D. Park, T. Theocharides, N. Vijaykrishnan, and C. R. Das. A low latency router supporting adaptivity for on-chip interconnects. In $D A C$ '05: Proceedings of the 42nd annual conference on Design automation, pages 559-564, 2005.

[11] A. Kodi, A. Sarathy, and A. Louri. Design of adaptive communication channel buffers for low-power area-efficient network-on-chip architecture. In $A N C S$ '07: Proceedings of the 3rd ACM/IEEE Symposium on Architecture for Networking and Communications Systems, pages 47-56, 2007.

[12] T. Krishna, A. Kumar, P. Chiang, M. Erez, and L.-S. Peh. NoC with near-ideal express virtual channels using global-line communication. In HOTI '08: Proceedings of the 2008 16th IEEE Symposium on High Performance Interconnects, pages 11-20, 2008.

[13] A. Kumar, L.-S. Peh, and N. K. Jha. Token flow control. In MICRO '08: Proceedings of the 2008 41st IEEE/ACM International Symposium on Microarchitecture, pages 342-353, 2008.

[14] A. Kumar, L.-S. Peh, P. Kundu, and N. K. Jha. Express virtual channels: towards the ideal interconnection fabric. In ISCA '0\%: Proceedings of the 34 th annual international symposium on Computer architecture, pages 150-161, 2007.

[15] J. Liu, L.-R. Zheng, and H. Tenhunen. A guaranteed-throughput switch for network-on-chip. In SOC '03: Proceedings of the International Symposium on System-on-Chip, pages 31-34, 2003.
[16] Z. Lu, M. Zhong, and A. Jantsch. Evaluation of on-chip networks using deflection routing. In GLSVLSI '06: Proceedings of the 16th ACM Great Lakes symposium on VLSI, pages 296-301, 2006.

[17] G. Michelogiannakis, J. Balfour, and W. J. Dally. Elastic buffer flow control for on-chip networks. In HPCA '09: Proceeding of the 15th International Symposium on High-Performance Computer Architecture, pages 151-162, 2009.

[18] G. Michelogiannakis, D. Pnevmatikatos, and M. Katevenis. Approaching ideal NoC latency with pre-configured routes. In NOCS '07: Proceedings of the First International Symposium on Networks-on-Chip, pages 153-162, 2007.

[19] T. Moscibroda and O. Mutlu. A case for bufferless routing in on-chip networks. SIGARCH Comput. Archit. News, 37(3):196-207, 2009.

[20] R. Mullins, A. West, and S. Moore. Low-latency virtual-channel routers for on-chip networks. In ISCA '04: Proceedings of the 31st annual International Symposium on Computer Architecture, page 188, 2004.

[21] C. Nicopoulos, D. Park, J. Kim, N. Vijaykrishnan, M. S. Yousif, and C. R. Das. ViChaR: A dynamic virtual channel regulator for network-on-chip routers. In MICRO '06: Proceedings of the 39th annual International Symposium on Microarchitecture, pages 333-344, 2006.

[22] E. Nilsson, M. Millberg, J. Oberg, and A. Jantsch. Load distribution with the proximity congestion awareness in a network on chip. In DATE '03: Proceedings of the conference on Design, Automation and Test in Europe, pages 1126-1127, 2003.

[23] E. Rijpkema, K. G. W. Goossens, A. Radulescu, J. Dielissen, J. van Meerbergen, P. Wielage, and E. Waterlander. Trade offs in the design of a router with both guaranteed and best-effort services for networks on chip. In DATE '03: Proceedings of the conference on Design, Automation and Test in Europe, page 10350, 2003.

[24] D. R. Rostislav, V. Vishnyakov, E. Friedman, and R. Ginosar. An asynchronous router for multiple service levels networks on chip. In $A S Y N C$ '05: Proceedings of the 11th IEEE International Symposium on Asynchronous Circuits and Systems, pages 44-53, 2005. 